\title{
Construction of smooth daily remote sensing time series data: a higher spatiotemporal resolution perspective
}

\author{
Zhuokun Pan ${ }^{1,2^{*}}$, Yueming $\mathrm{Hu}^{1,2}$ and Bin $\mathrm{CaO}^{3}$
}

\begin{abstract}
Research in time-series remote sensing data is receiving increasing attention. With the availability of relatively short repeat cycle and high spatial resolution satellite data, the construction and application of high spatiotemporal remote sensing time-series data is promising. In this paper, we proposed a method to construct complete spatial time series data, with Savitzky-Golay filter for smoothing and locally-adaptive linear interpolation for generating daily NDVI imagery. An IDL-based program was developed to achieve this goal. The China's HJ-1 A/B satellite data were employed for this remote sensing time series construction. The results demonstrated that: (1) This method can generate smooth continuous time series image data successfully based on irregularly short-revisit remote sensing data; (2) HJ-1 A/B NDVI time-series were demonstrated to be successful in monitoring crop phenology and hyperspectral analysis was successfully applied on $\mathrm{HJ}-1$ A/B time-series data to perform temporal endmember extraction. The IDL-based time-series construction program is generalizable for various kind of multi-temporal remote sensing data such as MODIS vegetation-index product. Discussion and concluding remarks are made to reveal the authors' perspective on higher spatial resolution time-series analysis in the remote sensing community.
\end{abstract}

Keywords: High spatiotemporal, Time-series, HJ-1 A/B, IDL program

\section{Introduction}

With the launch of high frequent remote sensing satellites and availability of data, time-series data derived from multi-temporal remote sensing images are receiving significant attention concerning the dynamics of regional vegetation growth, phenological crop identification, land use change detection, etc. [1-4]. Specifically, vegetation indices $(\mathrm{VI})$ products as time-series data have been widely employed in the remote sensing community. These data help us to understand the earth system and land-surface dynamics $[4,5]$. However, most of VI timeseries data was derived from low spatial resolution satellite platforms such as NOAA-AVHRR (Advanced Very High-Resolution Radiometer) instruments; EOS-MODIS (Moderate Resolution Imaging Spectro radiometer); and SPOT (Système Pour l'Observation de la Terre) VGT

\footnotetext{
* Correspondence: xyslz114@163.com

${ }^{1}$ Institute of Geoimformation Engineering, South China Agricultural

University, Guangzhou, China

${ }^{2}$ Key Laboratory of Construction Land Transformation, Ministry of Land and

Resources, Guangzhou, China

Full list of author information is available at the end of the article
}

product [6-10]. Higher spatiotemporal resolution images can deepen the understanding of land surface dynamics; generating consistent and comparative finer spatiotemporal time series imagery is therefore critical [11].

Several researchers have started to develop methods to increase the spatial resolutions to solve the trade-off between temporal and spatial resolution [12-15]. The term "data fusion" has been proposed to take advantage of scale and repeat cycle, examples just as the use of one scene of Landsat TM imagery to predict another time based on its relationship with high frequent MODIS imagery [16]. However, the performance of data fusion depends heavily on the sensitivity to both spatial heterogeneity and spectral inconsistency, which meant not all area are applicable [17]. Data fusion still rely on the availability of actual satellite images, and the quality of ingested remote sensing data. Even though it can be used to make synthetic images from multiple sources, these fused images cannot replace actual images [16].

With advancing technology for launching satellite constellations, multiple remote sensing satellites can be 
brought into orbit at low cost; with both radiometric and spatial consistency, these satellites bring new perspectives for earth observation $[4,15,18,19]$. One successful mission is Planet Labs' Remote Sensing Satellite System [20]. These small satellites provide high frequent revisiting cycle, which meets the requirement of daily observation.

Differed to idea of multi-source data fusion, the perspective of enhancing spatiotemporal resolution may be addressed by newly launched remote sensing satellites with real high spatial and temporal resolution data [15]. On the other hand, previous work done by Pan et al., they employ mono-source remote sensing data, a twoday-repeat HJ-1 A/B data, and developed daily time series construction method [3]. Similarly, Sun et al. redeveloped the TIMESAT program by modifying the adaptive smoothing and together with daily interpolation, that was aiming to generate daily $30 \mathrm{~m}$ Landsat time series [21].

Remote sensing time series data are commonly used in phenology monitoring. To facilitate the processing and analysis of time series, relevant researchers might have encountered with two computer programs: TIMESAT or SPIRITS [3, 18, 21-23]. Conventionally, MODIS data has been widely implemented in monitoring vegetation dynamics based on these two programs. However, these programs were designed to handle MODIS or SPOTVGT VI products that were organized at a fixed-day interval (e.g., 8 or 10 days composite). To date, higher resolution remote sensing satellites (e.g., Sentinel, RapidEye, Planet Labs) have become operational. However, few relevant studies were founded had adopted these short repeat-cycle and high spatial resolution data for time series analysis as the same way with MODIS. To construct a high spatiotemporal time series using these remote sensing data and make it applicable for TIMESAT or SPIRITS, specifically in phenology detection, one major problem is ensuring the continuousness and completeness of the time series dataset [3].

As such, up-to-date remote sensing satellite constellations provide sufficient images, which leads to the potential of constructing high spatiotemporal resolution time-series data. The motivation of writing this paper was to address an important topic by description of methodology together with a computer program that meets the common interest of facilitating time-series remote sensing data, specifically for up-to-date high spatial resolution satellite data.

\section{Data preparation}

China's HJ-1 A/B satellites data were employed for testing in this paper. Launched in 2008, HJ-1 A/B constellations are a new generation of small Chinese civilian remote sensing satellites [24]. The HJ-1 A/B satellites have two optical sensors to perform earth observation at 30-m resolution, with four bands covering the visible and near-infrared wavelength range. The double constellations constitute an observation network that covers China and its surrounding areas with a two-day repeat cycle. By taking advantage of its relatively high spatial resolution and frequent repeat cycle, some researchers demonstrated its potential in constructing dense timeseries data by data fusion with MODIS [11, 15].

The main task of this study is to make use of HJ-1 A/B for NDVI (normalized difference vegetation index) time-series data construction. Since the twoday-repeat $\mathrm{HJ}-1$ A/B satellites provide a considerable amount of images for time-series construction in our test site, we downloaded year-round HJ-1 A/B CCD images in 2012 for testing. There were 73 scenes available in 2012, and the images were cloud-free in the research area (Fig. 1 (a)). The site selected for testing HJ-1 A/B satellite data was Yangling, located in Guanzhong Plain of Shaanxi Province, China. This
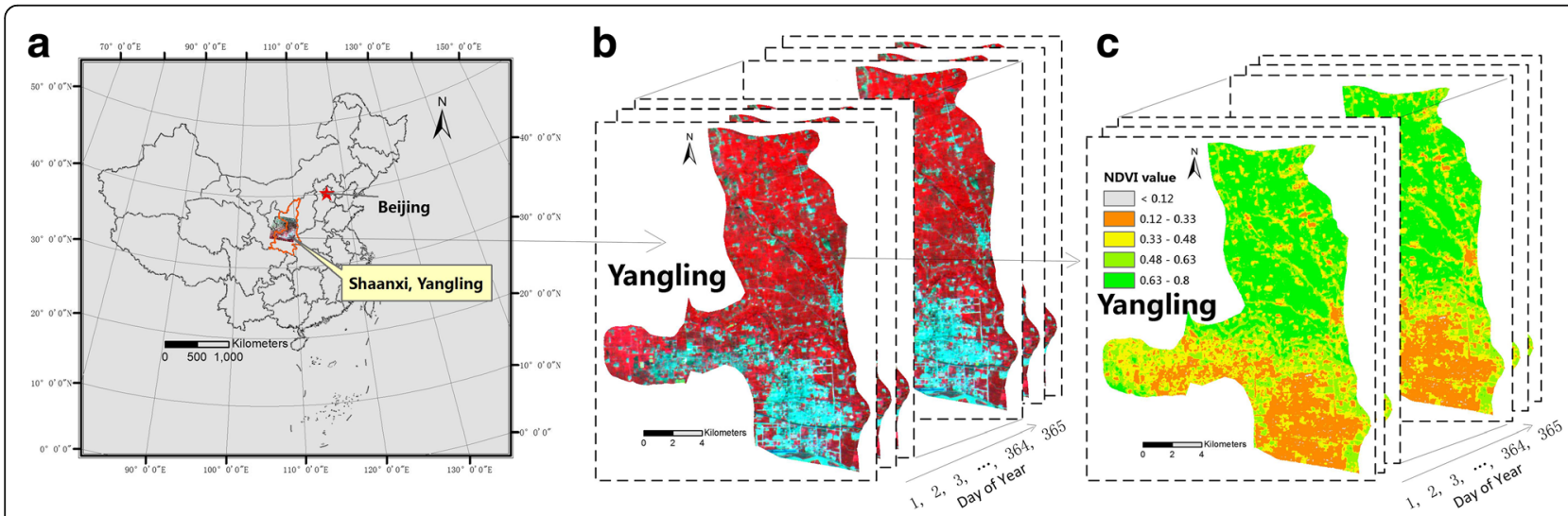

Fig. 1 Data preparation: (a) acquisition of HJ-1 A/B CCD images; (b) collection of subset images in the test site and coregistration; (c) calculation of NDVI and construction of the time-series dataset 
area has a double cropping system: the winter wheat is sowed in October and harvested in June; the summer corn is sowed in June and harvested in October. Production of these HJ-1 A/B NDVI are conventional as others remote sensing imagery, and the literature has summarized the procedure $[3,11,15]$. The NDVI images were layer stacked to construct a time-series dataset (Fig. 1 (b) and (c)).

\section{Methodology}

Satellite images captured by optical remote sensors usually contain noise due to weather conditions and changing solar illumination throughout the year $[25,26]$. When performing time-series smoothing, researchers are also reminded that maintenance of the original characteristics of the time-series profile is critical [25]. Just as with other high spatial resolution images, the tradeoff is that HJ-1 A/B time-series data is spaced irregularly in time. Signal processing techniques (e.g., wavelet and Fourier analysis) require the time-series data have regular, equidistant spacing $[27,28]$, hence they may not work well in the nonequidistantly spaced time-series data derived from $\mathrm{HJ}-1 \mathrm{~A} / \mathrm{B}$ satellites.

Moreover, remote sensing VI products such as MODIS and SPOT-VGT are organized in fixed-day intervals (e.g., 8 or 10 days composite). The processing methods available in time-series software (e.g., TIMESAT) do not function for unevenly distributed time-series; on the other hand, the function fitting or Fourier-based filters may be problematic when applied to irregular VI timeseries [29]. Therefore, in this section, we improved our method by first introduced the Savitzky-Golay (S-G) smoothing method. Then other smoothing methods were tested for comparison to prove its superiority. Finally, a missing-data interpolation was proposed to ensure regular spacing on a daily basis and generate daily NDVI images. IDL (Interactive Data Language) programming helps to achieve these goals.

\section{S-G smoothing method}

Time-series smoothing must be done to retrieve the essential shape of a curve. In this paper, the Savitzky-Golay (S-G) smoothing method was employed to facilitate the irregular spacing in HJ-1 A/B NDVI time-series. The S-G filter, also known as the least squares or digital smoothing polynomial, can be used to smooth a noisy signal [30]. The algorithm description can be described as below:

$$
g_{i}=\sum_{n=-n L}^{n R} c_{n} f_{i+n} / n
$$

where $f_{i}$ represents the original data value in the timeseries, and $g_{i}$ is the smoothed value, which is the linear combination of $c_{n}$ and $f_{i}$. Here, $\mathrm{n}$ is the width of the moving window to perform filtering, and $n L$ and $n R$ correspond to the left and right edge of the signal component. If $c_{n}$ is a constant defined as $c_{n}=1 /(n L+n R+1)$, then the S-G filtering becomes a moving average smoothing. The idea of S-G filtering is to find filtering coefficients $c_{n}$ that preserve higher moments. Therefore, in Eq.(2), $c_{n}$ is not a constant but a polynomial fitting function, typically quadratic or quartic. Then a leastsquares fit is solved, ranging from $n L$ to $n R$ to obtain $c_{n}$. For a specific dataset of a time series in a moving window, we define the fitting function as a quadratic polynomial for fitting a specific range of $f_{i}$ :

$$
c_{n}(t)=c_{1}+c_{2} t+c_{3} t^{2}
$$

where $t$ corresponds to the day of the year in NDVI time series. Therefore, the smoothed value $g_{i}$ can be obtained via Eq.(1). The result of S-G smoothing methods is shown in Fig. 2.

\section{Comparison between S-G and other methods}

Several commonly used methods for smoothing were tested as follows. The results could firmly demonstrate the S-G filter's superiority.

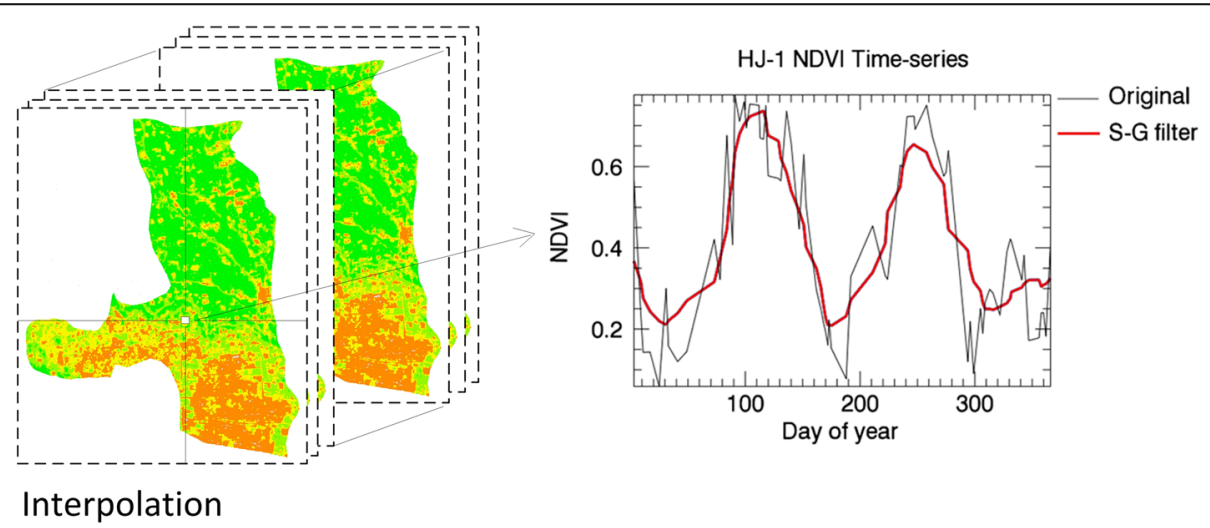

Fig. 2 Smoothing time-series data by S-G method 

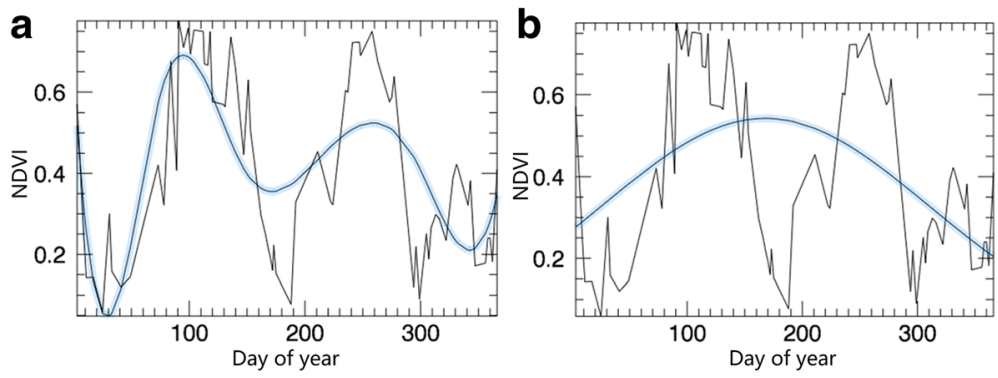

Fig. 3 Smoothing time-series data by function fitting with (a) a polynomial function and (b) a Gauss function

(1) Global function fitting

The SPLINE-curve fitting is a global function fitting to smooth discrete data. By forming a polynomial equation, a smoothed function curve is obtained to represent the discrete data. Likewise, other function fitting methods, such as using the asymmetric Gaussian model, have been adopted for fitting AVHRR-NDVI time-series data [1]. In this study, we applied a SPLINE-curve fitting, $y=a x^{3}+b x^{2}+c x+d$, and a Gaussian function fitting, $y=\mathrm{a} * e^{\left(-\frac{(x-\mathrm{b})^{2}}{c}\right)}$, on $\mathrm{HJ}-1 \mathrm{~A} / \mathrm{B}$ time series data for smoothing (Fig. 3). Figure 3 (a) appears to fit well but does not maintain the essential shape of the time-series trajectory; in Fig. 3 (b), the Gaussian function did not perform well in fitting a double cropping area which had two growth cycles over time. Apparently, as demonstrated, global function fitting methods are not suitable for unevenly spaced time-series data.

\section{(2)Signal denoising}

Viewing time series data as a signal, fast Fourier transformation (FFT) and a wavelet transform (WT) were adopted to handle the HJ-1 A/B time-series data. FFT and WT have already been applied on MODIS VI time series to retrieve a smoothed trajectory vegetation growth cycle [8]. We programmed the IDL-based fast Fourier transformation and the wavelet transform function to apply to $\mathrm{HJ}-1 \mathrm{~A} / \mathrm{B}$ time series data. However, as mentioned before, signal denoising (for FFT and WT) did not perform well for unevenly spaced time-series data (Fig. 4); no matter how the denoising parameters were set, such methods neither maintained an original shape nor preserved the original date in the time series.

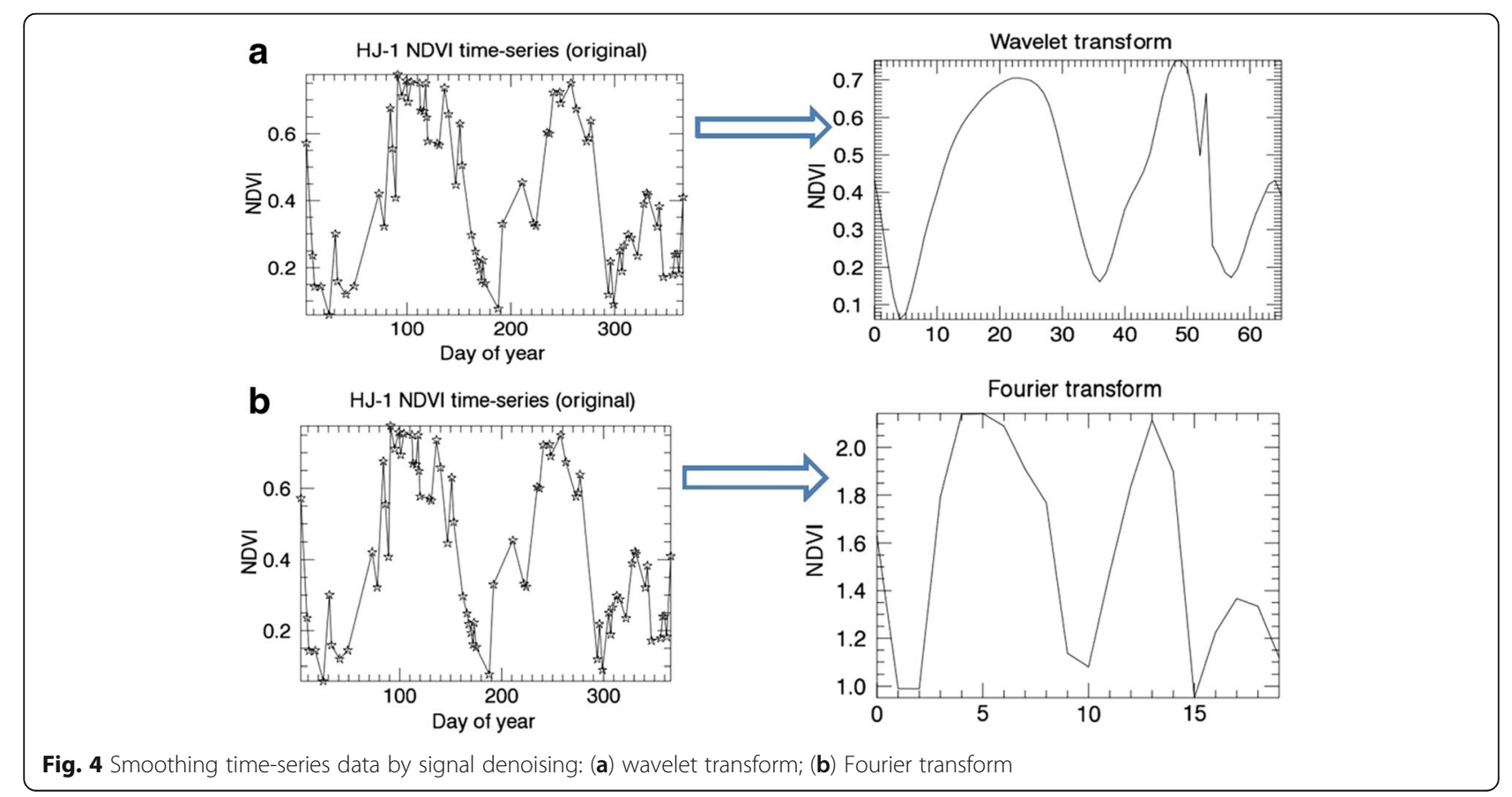




\section{(3)HANTS method}

HANTS (Harmonic Analysis of NDVI Time-Series) is a commonly used tool to smooth time-series remote sensing data (http://www.un-spider.org/links-andresour ces/gis-rs-software/hants\%C2\%A0harmonic\%C2\%A0analysis-of\%C2\%A0time\%C2\%A0series-nlrgdsc). HANTS can be used to remove cloud effects, smooth the data set, interpolate the missing data, and compress the data. Although the HANTS method could generate a pleasing looking time series, it has the same problem as FFT and WT when using the signal denoising method. For unevenly spaced HJ-1 A/B time-series data, HANTS tended to maintain the spatial completeness of a pixel profile (just as cloud removal), but to scarify the temporal characteristics. Moreover, the HANTS method did not preserve the original date in the time series; temporal characteristics revealing critical phenology details were drowned (Fig. 5).

\section{Generating daily NDVI images}

S-G filtering was employed to smooth HJ-1 A/B NDVI time-series data, to ensure a continuous and complete time-series dataset. A feasible approach was then proposed to ensure regular spacing on a daily basis. Linear interpolation is a simple interpolation method commonly used in mathematics and computer science. This paper developed a locally adaptive linear interpolation to generate missing data throughout the NDVI time series.
Missing data between two images can be generated by Eq.(3)-(4):

$$
\begin{aligned}
& \frac{N D V I-N D V I_{0}}{D O Y-D O Y_{0}}=\frac{N D V I_{1}-N D V I_{0}}{D O Y_{1}-D O Y_{0}} \\
& N D V I=N D V I_{0}+\left(N D V I_{1}-N D V I_{0}\right) * \frac{D O Y-D O Y_{0}}{D O Y_{1}-D O Y_{0}}
\end{aligned}
$$

where NDVI represents the missing day to be interpolated, and $N D V I_{1}$ and $N D V I_{O}$ represent the valid images used for the interpolation. Therefore, the NDVI between $N D V I_{O}$ and $N D V I_{1}$ can be treated as a linear relationship and then generated according to Eq. (4).

The smoothing performance and interpolation accuracy were evaluated by 1:1-line comparison as described in Fig. 6, both presented a goodness of fit.

\section{Code design}

Most commonly-used remote sensing software (e.g., ERDAS, ENVI) does not provide functionalities to manipulate time-series data; at present, TIMESAT and SPIRITS are also not designed to facilitate such relatively high spatial time series data. Additionally, an executable processing framework has not been found that allows researchers to obtain high spatiotemporal time-series dataset that meets their research demands. Although the methodology described above was easily achieved in operating a onedimensional array by most programming platforms, the
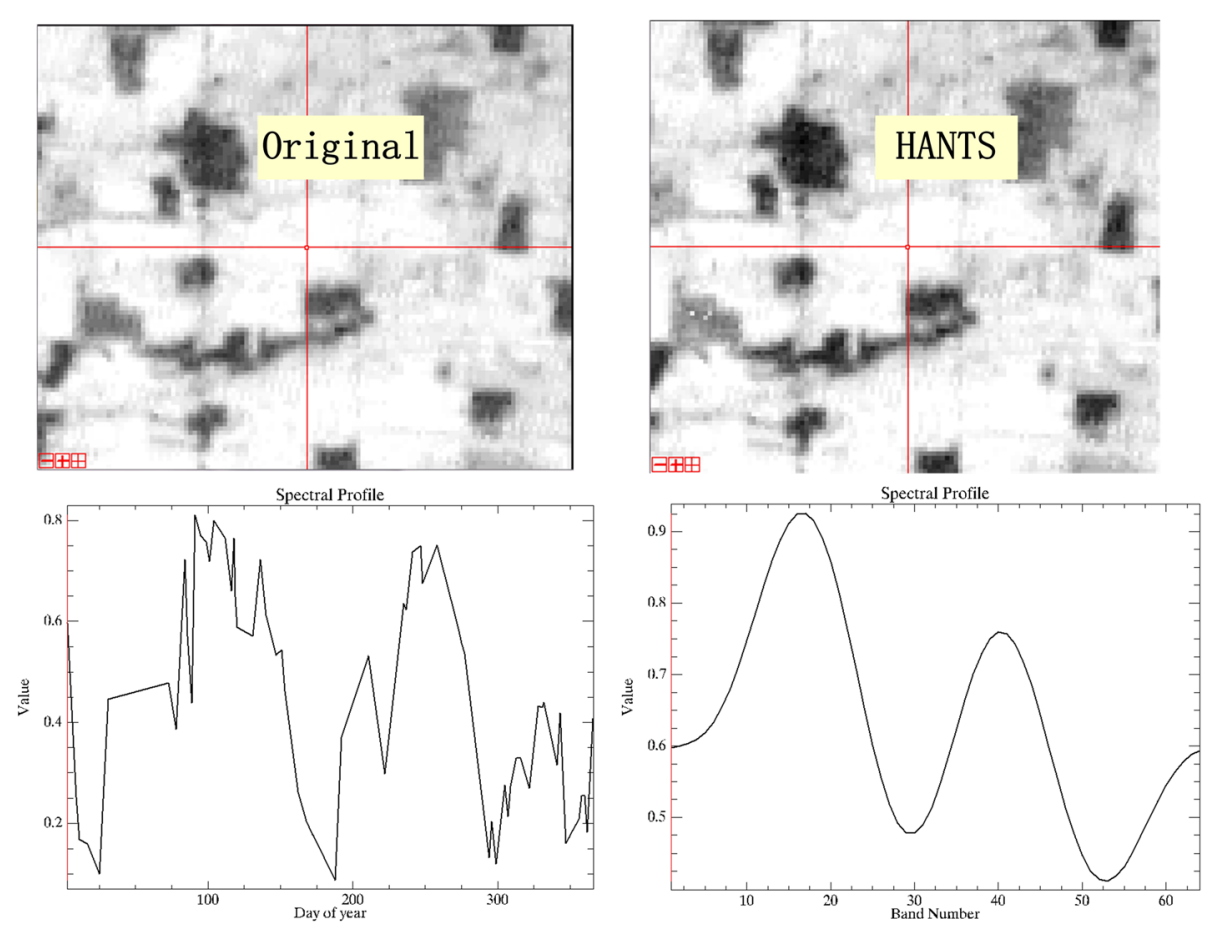

Fig. 5 Smoothing time-series data by HANTS method 

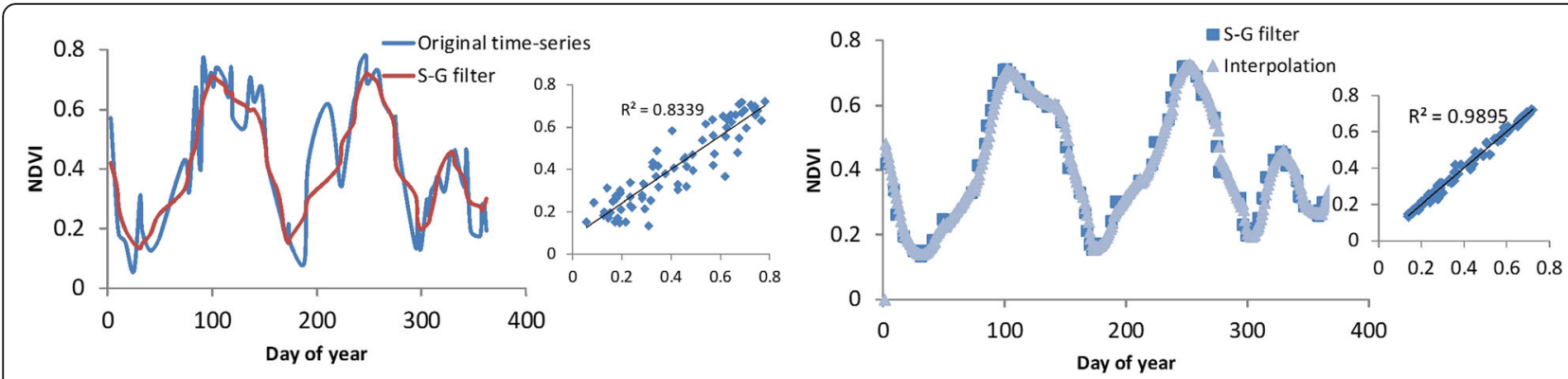

Fig. 6 Performance of time-series construction (a pixel sample from double-cropping land)

question raised here was how to perform smoothing and interpolation for time-series data with a three-dimensional array. IDL is an array-oriented language with numerous mathematical analysis and graphical display techniques, is ideal programming language for image data analysis, visualization, and cross-platform application development (http://www.harrisgeospatial.com/ProductsandTechnology/ Software/ENVI.aspx). Anyone who working with imagery or raster data has probably encountered ENVI software; its library routines are IDL-based functions and procedures. IDL programming is based on the ENVI function that is capable of operating remote sensing images; thus we developed a program to achieve filtering and interpolation of threedimension time-series data.

As introduced in S-G smoothing method section and Generating daily NDVI images section, this program mainly consists of two steps: time series filtering and image interpolation. This IDL-based program was developed with the IDL functions library, using Savitzky-Golay filtering and interpolation. The coding of this program was designed to treat time-series datasets as a three-dimension array by calling ENVI functions to manipulate individual images; the program loops pixel by pixel to extract the time-dimension to perform filtering and interpolation.

As described in section S-G smoothing method, the S-G filter will tend to minimize overall noise in NDVI timeseries to preserve the original trajectory. This IDL program requires users define the width of the moving window and degree of polynomial fitting in S-G filtering. The interpolation in the IDL program includes three commonly used methods: (1) simple linear interpolation, as described in Eq. (3)-(4); (2) least squares quadratic fit for each fourpoint neighborhood $(x[i-1], \quad x[i], \quad x[i+1], \quad x[i+2])$ surrounding the interval; and (3) SPLINE fit, which is a polynomial function fitting function for the four surrounding points. A user can use different interpolation methods to achieve optimal effect. The overall schematic of the functionality in this program is described in Fig. 7. Since the

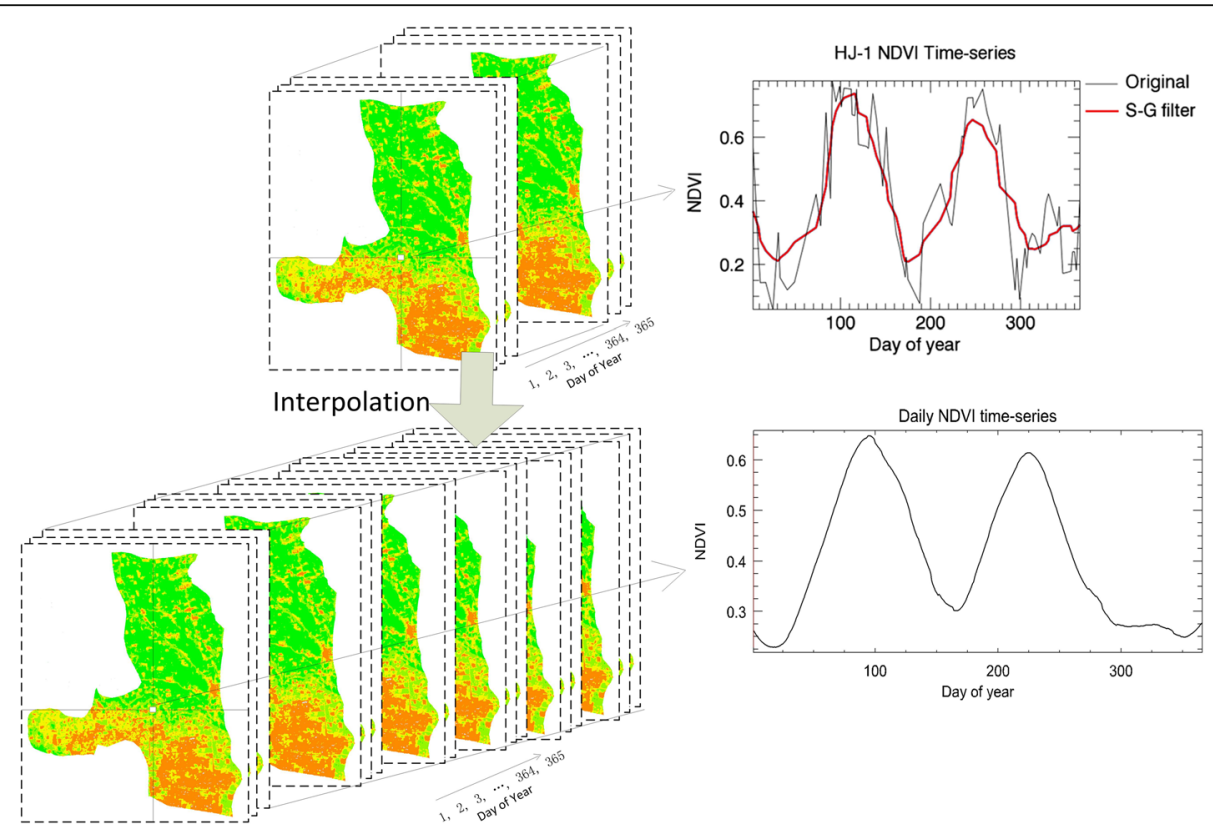

Fig. 7 Schematic of the smoothing and interpolation for NDVI images 
interpolation was applied locally in the time series within a defined interval, the result suggest that the essential shape of the NDVI trajectory was well maintained.

\section{Potential applications}

Because our method can handle equidistantly spaced high spatiotemporal remote sensing images to construct time series data, this paper provided further testing and potential applications.

\section{Extracting phenology}

Remote sensing data is particularly useful for detecting regional crop phenological characteristics [8]. HJ-1 A/B has a constellation of two satellites, which allows a twoday observation cycle; by the proposed methodology and the developed computer program, $\mathrm{HJ}-1 \mathrm{~A} / \mathrm{B}$ data can be used to construct a complete time-series dataset, making it possible to obtain key crop growth stages. Since the complete growth cycle of vegetation has been established with a daily interval, this study employed the TIMESAT program to extract the start and end of crop seasons from NDVI time series (Fig. 8).

Since phenology dynamic in remote sensing indicates the actual crop growth process in a pixel basis, these dynamic processes correspond directly to actual, ground-based phenological events, which provide indicators of climate vibrations; in addition, fine-scale crop seasonality reflects a spatial arrangement of agricultural activities. This study presented mapping of the start and end of crop seasons (measured in day-ofyear) for our test site in 2012 (Fig. 9). The results suggest that time-series data derived from $\mathrm{HJ}-1 \mathrm{~A} / \mathrm{B}$ satellites were applicable for extracting crop phenology, and the value distribution of phenological date (measured in day-of-year) was robust and convincing.

\section{Spectral analysis with spatial time-series data}

Time-series remote sensing data can be regarding as combinations of temporal endmembers in a temporal feature space where the dimensions represent different components of the time domain processes [31]. Hence, the time-series remote sensing data could be regarded as a hyperspectral-like dataset then to perform temporal endmembers extraction technique. Endmember extraction is the process of selecting a collection of pure signature spectra of the materials present in a hyperspectral image scene [32]. By analogy to spectral mixture analysis in spectral feature spaces, the temporal feature space conveys the spatiotemporal characteristics of ground substances. This study implemented the sequential maximum angle convex cone (SMACC) [33] spectral tool to extract temporal endmembers in NDVI time-series data.

As shown in Fig. 10, we extracted temporal endmembers representing several typical ground substances. The temporal trajectory in crop phenology is a good indicator for distinguishing cropping area and others land cover type. Additionally, as different types of vegetation have different phenologies, the cropping types are more easily discriminated. Aside from that, the time-domain phenomenon of VI time series reflects the process of crop growth and management level at different geographic locations; such information was quite important for agricultural productivity assessment.

\section{Test with MODIS EVI data}

Since ENVI/IDL-based programming provides convenient interactions for manipulating remote sensing images, our method is generalizable for multi-temporal remote sensing data to construct a smooth time-series dataset with

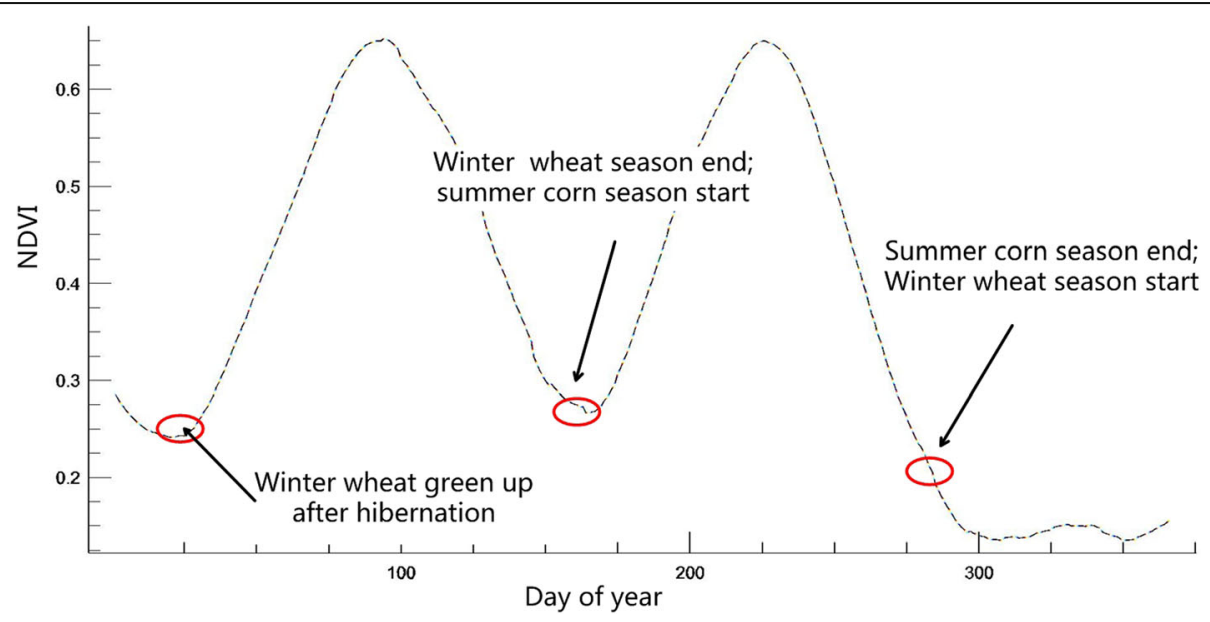

Fig. 8 Season start and end of double-cropping area in NDVI time series 


\section{Winter wheat green-up Winter wheat harvesting}
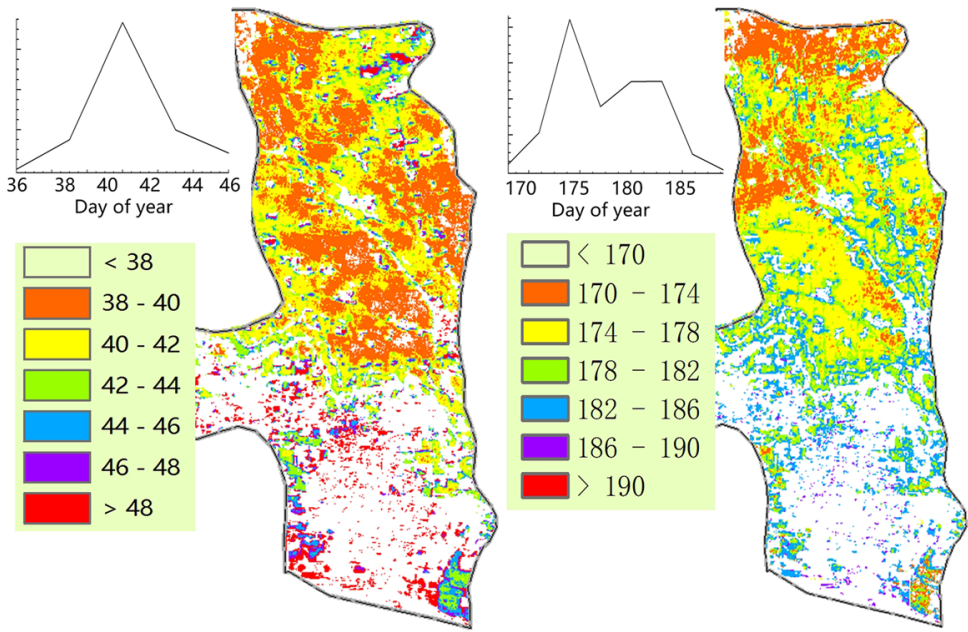

\section{Summer corn start}

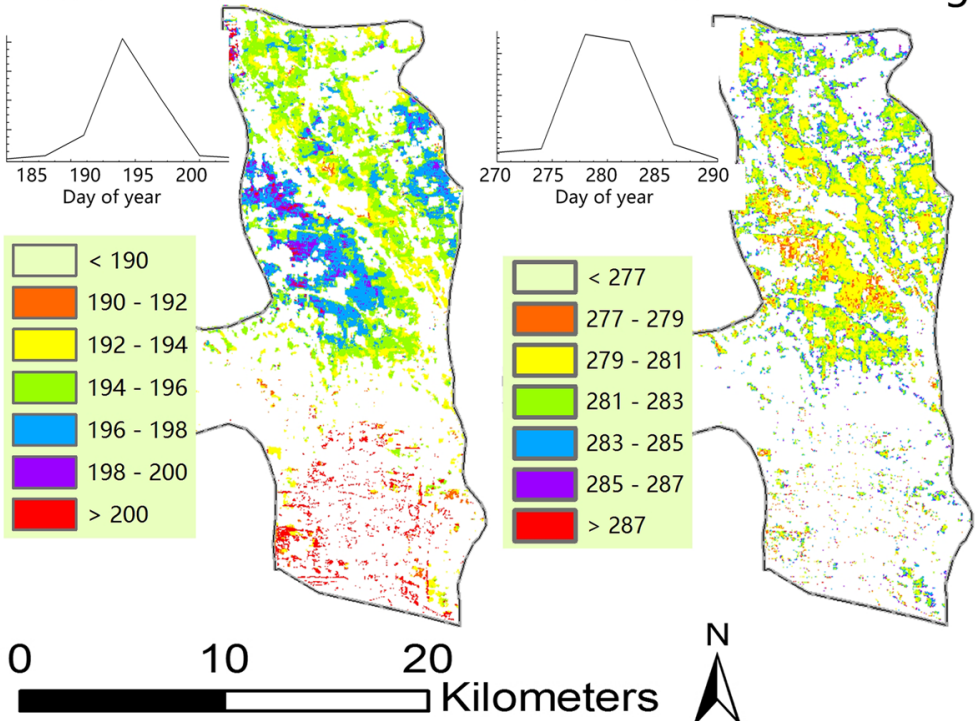

Fig. 9 Season start and end extraction for wheat-corn double-cropping land
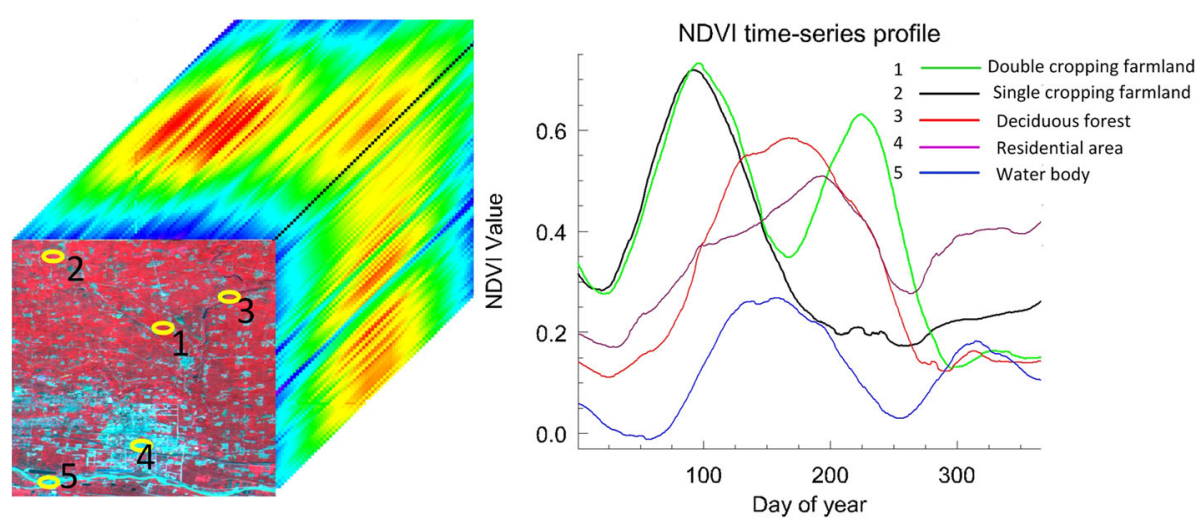

Fig. 10 Endmembers extraction with NDVI time-series data 
daily interpolation. For test purposes, 46 scenes of MODIS EVI images (8-day composite, $500 \mathrm{~m}$ resolution) were obtained within 1 year to construct a complete time series. The smoothing and interpolation result shown in Fig. 11 suggests that this program was capable of retrieving the essential trajectory of vegetation growth in the time dimension.

\section{Discussion}

Attributing to an explosive growth of data, research in time-series remote sensing data is receiving more and more attention, therefore, this study was conducted towards building higher spatial resolution, just as researchers do with the VI product derived from AVHRR, MODIS and SPOT-VGT. China's HJ-1 A/B remote sensing data has been successfully employed for constructing complete and applicable time-series data at 30-m resolution. However, very few research article reported using such relatively high spatiotemporal remote sensing data to construct a time-series dataset, and no processing software/tool is available for that purpose. Therefore, in addressing this important topic, a new method together with an IDL-based program for smoothing and interpolating time-series data was developed in this paper. Some remarks are proposed below.

When considering a high spatiotemporal time series, users should be acquainted with data availability in the study area and the expenses. In this paper, open-access HJ-1 A/B data were selected as cloud free, because the cloud-free NDVI data are relatively less affected by noise; the S-G filtering method works well in maintaining the essential shape of the NDVI trajectory, especially in accurately extracting vegetation phenology.

Other smoothing methods, like HANTS, are wellknown for time-series smoothing; however, HANTS tends to ensure spatial completeness but sacrifices important information in the time dimension [34]; meanwhile such drowned information is important in delineating vegetation growth. Likewise, alternative function fitting (sigmoid curve such as logistic model) or Fourier-based denoising were not suitable for implementation because their strict shape-matching was problematic in handling the unevenly spaced time-series data derived from $\mathrm{HJ}-1 \mathrm{~A} / \mathrm{B}$ satellites. Using those methods may exaggerate high fluctuations in time-series data, making the result inconvincible. A comparison of smoothing methods was tested in section Comparison between S-G and other methods. Particularly, when time series data is constructed for phenology detection, a smoothing method is to be cautiously considered [25]. Methodologies employed in this paper for smoothing and interpolating were not aimed at producing the most visually pleasing result, but the most accurate. More comments and discussions can be referred to in the literature $[25,29,35,36]$.

Several articles indicate the Sentinel-2 A/B data is promising $[11,18]$. With their short-term global revisiting cycle and $10-\mathrm{m}$ resolution, they will play an additional role in high spatiotemporal remote sensing of time series data. In addition, current satellite constellations for commercial purposes, such as RapidEye and Planet Labs, should be considered for building a time series, probably the underlying value within time series analysis is truly need for public.

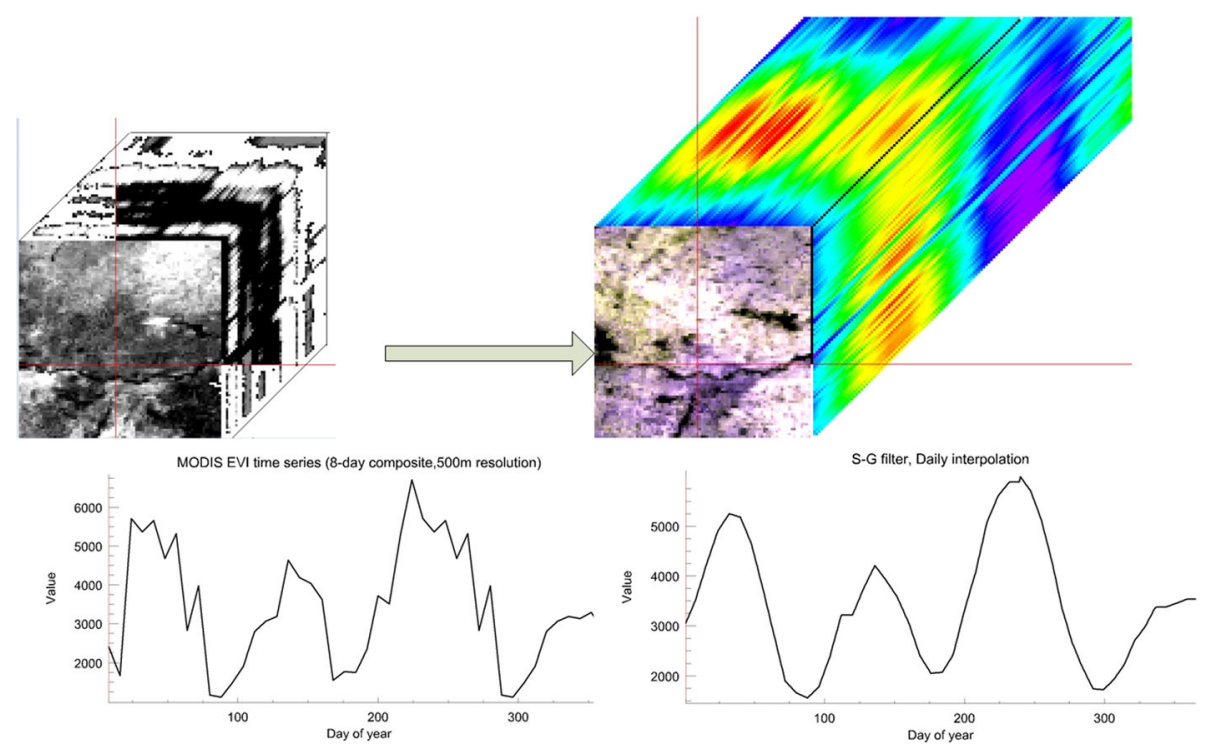

Fig. 11 A test with MODIS EVI product 


\section{Conclusions and perspectives}

The motivation of this paper was to provide a new method and a computer program that facilitates the construction of time-series remote sensing data with generalizable, potential, and practical applications, specifically for up-todate high spatial resolution satellite data. To achieve this goal, this study presented comprehensive processing procedures to construct HJ-1 A/B NDVI time series: the Savitzky-Golay smoothing method was employed first to reduce noise components from the original curve to retrieve the original shape of the time-series profile; then, a locally adaptive linear interpolation was employed to generate daily NDVI based on the available imageries. Afterward, the IDL-based program was developed to fulfill these procedures.

Our method was able to produce high-quality NDVI time series and might advance its application in various fields of study. As application cases introduced in Potential applications section, firstly application of HJ1 A/B NDVI time-series data in a fine-scale phenology characterization was presented; secondly, we extracted typical endmembers from the time-series data that represented spatiotemporal characteristics of ground substances, indicating the potential of using hyperspectral analysis techniques with time-series remote sensing data. Finally, the MODIS EVI data was used for testing; the result suggested that this program is generalizable for most time-series remote sensing data. As a primary test, based on the result above, we believed that higher resolution time series remote sensing data would make it much valuable than conventional MODIS or SPOT-VGT, making civilian acceptable and accessible to such techniques in real life. Since high-frequency remote sensing data will no longer be restrained to the medium and low resolution domains, the importance of time-series remote sensing data had been recognized, and higher spatial resolution will add on to these. Researchers should be encouraged to advance its applications in other disciplines. Constructing high spatiotemporal time series data requires a considerable amount of multi-temporal images. Researchers may be concerned about the cost, data acquisition and preprocessing. Currently, programs and tools for satellite image processing are facing technical challenges with upcoming satellite sensors with increasing spatial and temporal resolution. There is an urgent need to develop standards for data processing of flow and analysis systems, which would allow fast data processing to facilitate higher-magnitude time series data $[3,18]$.

\section{Endnotes}

${ }^{1}$ https://github.com/panzhuokun/Time-series-remotesensing-construction-IDL-code-.git

\section{Acknowledgements}

Our research was supported by the National Science Foundation of China (Grant NO. U1301253), and the International Postdoctoral Exchange Fellowship Program 2017 (Grant NO. 20170029).

\section{Availability of data and materials}

Readers who are interested in using the methods to process time series remote sensing data, the source code of this project can be viewed and checked out from a Github repository, ${ }^{1}$ and it is written in IDL. The source code is also available within each release. Questions are welcomed through E-mail contact: xyslz114@sina.com (panzhuokun).

\section{Authors' contributions}

ZP originally designed and conduct the research, and wrote this manuscript; $\mathrm{YH}$ is Pan's supervisor who provides additional funding for his research; BC had tested the program and made a revision before submitting. All authors read and approved the final manuscript.

\section{Competing interests}

The authors declare that they have no competing interests.

\section{Publisher's Note}

Springer Nature remains neutral with regard to jurisdictional claims in published maps and institutional affiliations.

\section{Author details}

${ }^{1}$ Institute of Geoimformation Engineering, South China Agricultural University, Guangzhou, China. ${ }^{2}$ Key Laboratory of Construction Land Transformation, Ministry of Land and Resources, Guangzhou, China. ${ }^{3}$ College of Marine Science, Shanghai Ocean University, Shanghai, China.

Received: 20 July 2017 Accepted: 13 September 2017

Published online: 05 October 2017

\section{Reference}

1. Jönsson P, Eklundh L. Seasonality extraction by function fitting to time-series of satellite sensor sata. IEEE Trans. Geosci. Remote Sens. 2002;40(8):1824-32.

2. Fensholt R, Proud SR. Evaluation of Earth Observation based global long term vegetation trends - Comparing GIMMS and MODIS global NDVI time series. Remote Sens Environ. 2012;119:131-47. doi:10.1016/j.rse.2011.12.015.

3. Pan Z, Huang J, Zhou Q, Wang L, Cheng Y, Zhang H, et al. Mapping crop phenology using NDVI time-series derived from HJ-1 A/B data. Int J Appl Earth Obs Geoinf. 2015;34:188-97. doi:10.1016/j.jag.2014.08.011.

4. Künzer C, Stefan D, Wolfgang W. Remote Sensing Time Series-Revealing Land Surface Dynamics. Springer. 2015

5. Guyet T, Nicolas H. Long term analysis of time series of satellite images. Pattern Recogn Lett. 2016;70:17-23. doi:10.1016/j.patrec.2015.11.005.

6. Jakubauskas ME, Legates DR, Kastens JH. Crop identification using harmonic analysis of time-series AVHRR NDVI data. Comput Electron Agric. 2002;37:127-39.

7. Reed BC, Brown JF, VanderZee D, Loveland TR, Merchant JW, Ohlen DO. Measuring phenological variability from satellite imagery. J Veg Sci. 1994;5:703-14.

8. Sakamoto T, Yokozawa M, Toritani H, Shibayama M, Ishitsuka N, Ohno H. A crop phenology detection method using time-series MODIS data. Remote Sens Environ. 2005;96(3-4):366-74. doi:10.1016/j.rse.2005.03.008.

9. Verbeiren S, Eerens H, Piccard I, Bauwens I, Van Orshoven J. Sub-pixel classification of SPOT-VEGETATION time series for the assessment of regional crop areas in Belgium. Int J Appl Earth Obs Geoinf. 2008;10(4):486-97. doi:10. 1016/j.jag.2006.12.003.

10. Zhang $X$, Friedla MA, Schaaf CB, Strahler AH, Hodges JCF, Gao F, et al. Monitoring vegetation phenology using MODIS. Remote Sens Environ. 2003; 84:471-5.

11. Bian J, Li A, Wang Q, Huang C. Development of Dense Time Series 30-m Image Products from the Chinese HJ-1A/B Constellation: A Case Study in Zoige Plateau, China. Remote Sens. 2015;7(12):16647-71. doi:10.3390/ rs71215846.

12. Gao F, Masek J, Schwaller M, Hall F. On the blending of the Landsat and MODIS surface reflectance: Predicting daily Landsat surface reflectance. IEEE Trans. Geosci. Remote Sens. 2006;44:2207-18.

13. Schmidt M, Udelhoven $T$, Gill T, Röder A. Long term data fusion for a dense time series analysis with MODIS and Landsat imagery in an Australian Savanna. J. Appl. Remote. Sens. 2012;6(1):063512. 
14. Wu M, Niu Z, Wang C, Wu C, Wang L. Use of MODIS and Landsat time series data to generate high-resolution temporal synthetic Landsat data using a spatial and temporal reflectance fusion model. J Appl Remote Sens. 2012;6(1):063507.

15. Wu M, Zhang X, Huang W, Niu Z, Wang C, Li W, et al. Reconstruction of Daily 30 m Data from HJ CCD, GF-1 WFV, Landsat, and MODIS Data for Crop Monitoring. Remote Sens. 2015;7(12):16293-314. doi:10.3390/rs71215826.

16. Gao F, Hilker T, Zhu X, Anderson M, Masek J, Wang P, et al. Fusing Landsat and MODIS Data for Vegetation Monitoring. IEEE Geoscience and Remote Sensing Magazine. 2015;3(3):47-60.

17. Kong F, Li X, Wang H, Xie D, Li X, Bai Y. Land Cover Classification Based on Fused Data from GF-1 and MODIS NDVI Time Series. Remote Sens. 2016; 8(9):741. doi:10.3390/rs8090741.

18. Rembold F, Meroni M, Urbano F, Royer A, Atzberger C, Lemoine G, et al. Remote sensing time series analysis for crop monitoring with the SPIRITS software: new functionalities and use examples. Front Environ Sci 2015;3. doi:10.3389/fenvs.2015.00046.

19. Sandau R, Brieß K, D’Errico M. Small satellites for global coverage: Potential and limits. ISPRS J Photogramm Remote Sens. 2010;65(6):492-504. doi:10.1016/j.isprsjprs.2010.09.003.

20. Marshall W, Boshuizen C. Planet Labs' Remote Sensing Satellite System. Proceedings of the AIAA/USU Conference on Small Satellites. 2013.

21. Sun L, Gao F, Anderson M, Kustas W, Alsina M, Sanchez L, et al. Daily Mapping of $30 \mathrm{~m} \mathrm{LAl}$ and NDVI for Grape Yield Prediction in California Vineyards. Remote Sens. 2017;9(4):317. doi:10.3390/rs9040317.

22. Eerens $H$, Haesen D, Rembold F, Urbano F, Tote C, Bydekerke L. Image time series processing for agriculture monitoring. Environ Model Softw. 2014;53: 154-62. doi:10.1016/j.envsoft.2013.10.021.

23. Jönsson P, Eklundh L. TIMESAT—a program for analyzing time-series of satellite sensor data. Comput Geosci. 2004;30(8):833-45. doi:10.1016/j.cageo. 2004.05.006

24. Wang Q, Wu C, Li Q, Li J. Chinese HJ-1A/B satellites and data characteristics. Science China (Earth Sciences edition). 2011;53(51):51-7. doi:10.1007/s11430010-4139-0.

25. Hird JN, McDermid GJ. Noise reduction of NDVI time series: An empirical comparison of selected techniques. Remote Sens Environ. 2009:113(1):248-58. doi:10.1016/j.rse.2008.09.003.

26. Sakamoto T, Wardlow BD, Gitelson AA, Verma SB, Suyker AE, Arkebauer TJ. A Two-Step Filtering approach for detecting maize and soybean phenology with time-series MODIS data. Remote Sens Environ. 2010;114(10):2146-59. doi:10.1016/j.rse.2010.04.019.

27. Baisch S, Bokelmann Gt HR. Spectral analysis with incomplete time series: an example from seismology. Comput Geosci. 1999;25:739-50.

28. Schulz M, Stattegger K. Spectrum: spectral analysis of unevenly spaced paleoclimatic time series. Comput Geosci. 1997;9(23):929-45.

29. Cong N, Piao S, Chen A, Wang X, Lin X, Chen S, et al. Spring vegetation greenup date in China inferred from SPOT NDVI data: A multiple model analysis. Agric For Meteorol. 2012;165:104-13. doi:10.1016/j.agrformet.2012.06.009.

30. Savitzky A, Golay MJE. Smoothing and differentiation of data by simplified least Squares procedures. Anal Chem. 1964;36(8):1627-39.

31. Small C. Spatiotemporal dimensionality and Time-Space characterization of multitemporal imagery. Remote Sens Environ. 2012;124:793-809. doi:10. 1016/j.rse.2012.05.031.

32. Plaza A, Martín G, Plaza J, Zortea M, Sánchez S. Recent Developments in Endmember Extraction and Spectral Unmixing. Optical Remote Sensing. 2011:235-67. doi: 10.1007/978-3-642-14212-3_12.

33. Gruninger J, Ratkowski AJ, Hoke ML, Lewis PE. The sequential maximum angle convex cone (SMACC) endmember model. Algorithms and Technologies for Multispectral, Hyperspectral, and Ultraspectral Imagery (Proceedings of SPIE). 2004;5425:1-14. doi:10.1117/12.543794.

34. $X u$ Y, Shen $Y$. Reconstruction of the land surface temperature time series using harmonic analysis. Comput Geosci. 2013;61:126-32. doi:10.1016/j. cageo.2013.08.009.

35. Bradley BA, Jacob RW, Hermance JF, Mustard JF. A curve fitting procedure to derive inter-annual phenologies from time series of noisy satellite NDVI data. Remote Sens Environ. 2007;106(2):137-45. doi:10.1016/j.rse.2006.08.002

36. Julien Y, Sobrino JA. Comparison of cloud-reconstruction methods for time series of composite NDVI data. Remote Sens Environ. 2010;114(3):618-25. doi:10.1016/j.rse.2009.11.001.

\section{Submit your manuscript to a SpringerOpen ${ }^{\circ}$ journal and benefit from:}

- Convenient online submission

- Rigorous peer review

- Open access: articles freely available online

- High visibility within the field

Retaining the copyright to your article

Submit your next manuscript at $>$ springeropen.com 